\title{
Landschaftsmaßnahmen auf den Gemeinschaftsweiden im Südschwarzwald
}

\section{Einleitung}

Zwischen Freiburg und Basel liegt im Südwestschwarzwald ein ca. $800 \mathrm{~km}^{2}$ großes Gebiet, in dem die gemeinschaftliche Weide auch heute noch die vorherrschende Form der landwirtschaftlichen Bodennutzung ist (Fig. 1).

Etwas über $50 \%$ der landwirtschaftlich genutzten Flächen sind Gemeinschaftsweiden, wovon Allmendweiden (heute Gemeindeweiden genannt) den weitaus

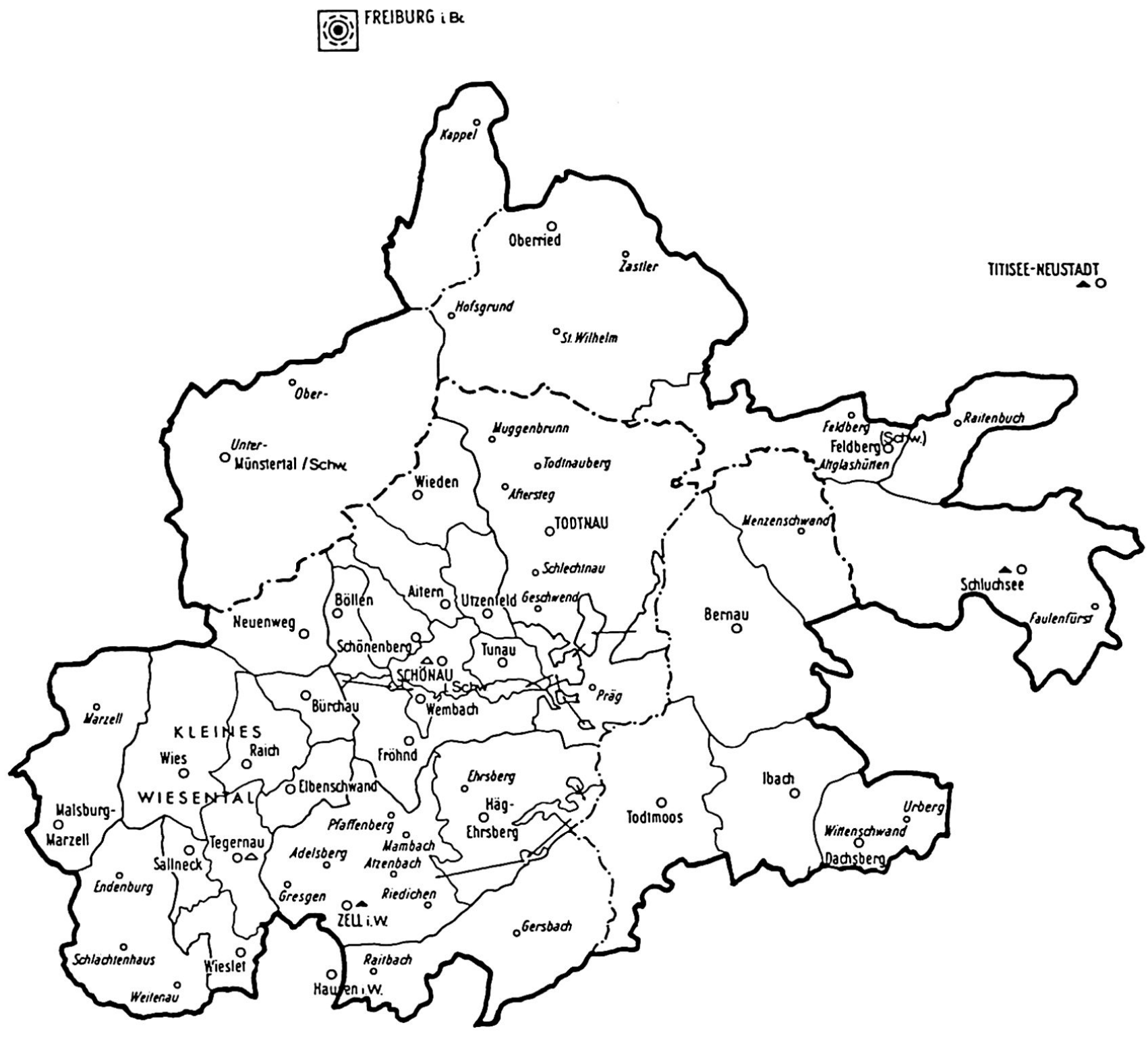

Fig.1 Gebiet der Gemeinschaftsweiden im Südschwarzwald

Dr. Konrad Müller, Regierungspräsidium Freiburg Erbprinzenstr. 2, D-7800 Freiburg i. Brg. 
größten Teil ausmachen. Kleinere Anteile haben die privaten Gemeinschafts- und Genossenschaftsweiden. Schon vor über 50 Jahren wurde vom Staat eine besondere Dienststelle für die Beratung und $\mathrm{Be}$ treuung der Gemeinschaftsweiden geschaffen, die Staatliche Weideinspektion mit Sitz in Schönau, eine Außenstelle des Regierungspräsidiums Freiburg.

Wir sprechen von Landschaftspflege und nicht von Brachlandpflege, weil

- in Extensivweidegebieten der Übergang zwischen noch genutzten und nicht mehr genutzten Flächen häufig fließend ist. Die gleiche Maßnahme, etwa das Roden von Gebüsch, kann deshalb im einen Fall zur Weidebewirtschaftung gehören, im anderen Fall jedoch ganz der Landschaftserhaltung dienen

- wir folglich eine wenn auch nur extensive Beweidung einer möglichst großen Fläche anstreben und nicht den Rückzug auf die kleinere, entsprechend intensiver bewirtschaftete Fläche.

Landschaftspflege durch das Weidetier steht also an erster Stelle, die im folgenden zu besprechenden weiteren Maßnahmen sind Ergänzung, vereinzelt natürlich auch Ersatz der Beweidung.

\section{Beweidung}

Der Rückgang der Rinderauftriebe, der wichtigsten Tierart, in den sechziger Jahren war für uns eine große Sorge und der Anlaß, uns um die Wiederbelebung der in der Kriegs- und Nachkriegszeit zwangsverfügten Schafbeweidung zu bemühen. Sie sehen, daß das gelungen ist: Heute werden rund 1500 ha der Gemeinschaftsweiden durch über 8000 Schafe genutzt (Tab. 1).

\section{Tab.1 Auftriebe auf die Gemeinschaftsweiden}

\begin{tabular}{lcrr} 
Jahr & Rinder & Schafe & Ziegen \\
\hline 1951 & 7956 & 2553 & 1974 \\
1961 & 8330 & 77 & 202 \\
1963 & 8038 & 67 & 115 \\
1965 & 7392 & 36 & 62 \\
1967 & 7813 & 44 & 38 \\
1969 & 7548 & 115 & 16 \\
1971 & 8083 & 153 & 15 \\
1981 & 11105 & 8614 & 103 \\
\hline
\end{tabular}

Dank agrarpolitischer Maßnahmen, vor allem durch die Ausgleichszulage, sind aber auch die Rinderauftriebe wieder stark gestiegen, so daß die Weiden heute meist voll belegt sind und vielfach die Wiedereinbeziehung ehemaliger, inzwischen verbuschter Weideflächen angestrebt wird.
Die früher sehr umfangreiche Ziegenhaltung wird dagegen keine Bedeutung mehr erlangen. Der Anstieg der Zahlen zwischen 1971 und 1981 ist auf einen von uns durchgeführten Landschaftspflegeversuch mit Ziegen zurückzuführen.

\section{Zusätzliche Maßnahmen}

Die Ergänzungs- oder Ersatzmaßnahmen dienen der Zurückdrängung von drei Pflanzengruppen:

- Gebüschaufwuchs, Stockausschlag und Gehölzanflug, insgesamt «Verhurstung» genannt; die Bekämpfung heißt deshalb «Enthursten» (ca. $1200 \mathrm{ha})$,

- kleine Ginsterarten (vorwiegend Genista anglica, tinctoria und sagittalis), die auf an sich guten Flächen lästige Weideunkräuter sein können (ca. 400 ha),

- Farn (fast ausschließlich Pteridium aquilinum), der sich dort, wo er nicht laufend bekämpft wurde, zu geschlossenen, alle übrigen Pflanzen unterdrükkenden Beständen entwickelt hat (ca. 600 ha).

Als Bekämpfungsmaßnahmen werden eingesetzt:

- Handarbeit

- Chemische Mittel

- Maschinen

Als Handarbeitsmaßnahme wird heute fast nur noch Gehölzaufwuchs enthurstet, das früher verbreitete Farnmähen spielt aus arbeitswirtschaftlichen und Kostengründen keine Rolle mehr.

\section{Tab. 2 Handarbeit (Enthursten);}

in Regie der Gemeinden: Umfang und Kosten im Jahr 1982

Schwankungsbreite der Gesamtkosten von 300.00 bis 1800.00 DM/ha

1.

Nutzungsberechtigte

Landwirte: $\quad 76,2$ ha

mittlere Gesamtkosten $\quad 1200.00 \mathrm{DM} / \mathrm{ha}$

Zuschuß Land

Baden-Württemberg $\quad 50 \%$ (Naturschutzfonds)

2.

Spontane Aktionen*:

3.

Patenschaften örtlicher

Vereine:

ca. 100 ha

keine Kostenermittlung

Anerkennungsgebühr

von Gemeinde

50.00 bis $100.00 \mathrm{DM} / \mathrm{ha}$

* Private Umweltschutzorganisationen, Bürgerinitiativen usw. 
Die Enthurstungsarbeiten werden teilweise von den Gemeinden, in zunehmenden Umfang aber auch von einem vor 2 Jahren gegründeten Weide- und Landschaftspflegezweckverband organisiert, dem alle $\mathrm{Ge}$ meinschaftsweiden angeschlossen sind.

Nach unseren langjährigen Erfahrungen schwanken die Kosten der in Regie der Gemeinde durchgeführten Enthurstungsarbeiten in dem weiten Bereich zwischen 300.- und 1800.- DM/ha.

Die Weidenutzungsberechtigten müssen zur Bewirtschaftung der Flächen 8-10 Stunden «Pflichtarbeit»/ Stück Vieh leisten. Die hier aufgeführten 76 ha Enthurstungen sind darüber hinaus erbrachte Leistungen, die in Form von Arbeitsnachweisen der Gemeinde in Rechnung gestellt und von dieser bezahlt werden.

Spontane Aktionen gab es im vergangenen Jahr keine. Mehr versprechen wir uns von LandschaftspflegePatenschaften für bestimmte Flächen durch örtliche Vereine. Dieses Verfahren ist in einer Gemeinde gut angelaufen und findet zunehmend Nachahmung. Die Gemeinde zahlt dafür einen kleinen Beitrag in die Vereinskasse. Zur Zeit wird noch geprüft, ob sich das Land an den Patenschaften finanziell ebenfalls beteiligen wird.

Die in der Regie des Zweckverbandes durchgeführten Enthurstungen sind schon deshalb wesentlich teurer, weil der Landschaftspflegetrupp vorwiegend in schwierigem Gelände und bei stärkerer Verbuschung eingesetzt wird.

Die Aktion ist auf Bitten der Arbeitsverwaltung im Jahr 1976 angelaufen, die damals nach Möglichkeiten suchte, im Rahmen des Arbeitsbeschaffungsprogramms Arbeitslose unterzubringen. Seit 1979 können auch Wehrdienstverweigerer ihren Zivildienst bei uns ableisten.

\section{Tab. 3 Handarbeit (Enthursten);}

in Regie des Zweckverbandes: Umfang und Kosten im Jahr 1982

Schwankungsbreite der Gesamtkosten von 1000.00 bis 4000.00 DM/ha

\begin{tabular}{ll}
\hline $\begin{array}{l}\text { Landschaftspflegetrupp: } \\
\text { (durchschnittl. 9 Mann) }\end{array}$ & rund 100 ha \\
mittlere Gesamtkosten: & $2369.00 \mathrm{DM} / \mathrm{ha}$ \\
\hline
\end{tabular}

Die über den Zweckverband laufenden Maßnahmen sind für die Gemeinden in finanzieller Hinsicht sehr günstig. Ihre tatsächliche Kostenbeteiligung ist aber meist höher, denn

- fast immer ist ein ortskundiger Mann zur Aufsicht oder als Vorarbeiter notwendig,
- das Verbrennen der vom Pflegetrupp zusammengetragenen Holz- und Reisighaufen obliegt im folgenden Jahr der Gemeinde oder den Nutzungsberechtigten.

Die heute sehr umstrittenen chemischen Mittel werden nur in ganz geringem Umfang (stets unter $0,5 \%$ der Gesamtfläche je Jahr) verwendet. Da wir selbst bestrebt sind, möglichst von der Chemie wegzukommen, wurde 1981 und 1982 gegen Gebüsch und Ginster nicht mehr gespritzt. Beim Adlerfarn sehe ich dagegen zur Zeit noch keine Möglichkeit, ganz von der chemischen Bekämpfung wegzukommen, da die bisher einzige Alternative - 6-7 Jahre lang zweimal pro Jahr mähen - heute nicht mehr durchführbar ist.

So problematisch die Chemie ist, so ist sie, richtig angewandt, nicht nur wirksam, sondern auch kostengünstig. Da gegen Gebüsch nur punktuell, also nicht flächig gespritzt wurde, lagen die Kosten kaum einmal über 400.- DM/ha. Hinzu kamen bei stärkerem Holz und dichterem Bewuchs etwa gleich hohe Kosten für das Abräumen.

Der Weide- und Landschaftspflegezweckverband hat jetzt eine Landschaftspflegemaschine bestellt, die in diesem Sommer erstmals auf größeren Flächen gegen Gebüsch- und Stockausschlag und zum Mulchen eingesetzt werden soll. Zum Maschineneinsatz kann ich also nur die von uns kalkulierten voraussichtlichen Kosten angeben.

\section{Tab. 4 Voraussetzungen und Grenzen des Maschineneinsatzes zur Landschaftspflege}

1.

Hangneigung: bis durchschnittlich $50 \%$

(dabei Abweichungen an (ca. $26^{\circ}$ )

Einzelpunkten bis $+30 \%$

[ca. 17\%] möglich)

2.

Hangfestigkeit:

Bodenart und -zustand Feuchtigkeit Bewuchs

3. Arbeitsgeschwindigkeit: $\operatorname{maximal} 7,5 \mathrm{~km} / \mathrm{h}$

4. Kraftbedarf:

14-35 PS/m Arbeitsbreite Schlegelmulchgerät (Arbeitsbreite der Geräte: $\quad 1,50 \mathrm{~m}$ bis $2,40 \mathrm{~m}$ ) 5. Holzstärke: $\quad$ sinnvoll bis maximal $6 \mathrm{~cm}$ Durchmesser 
Durch zahlreiche Versuche wissen wir allerdings ziemlich gut Bescheid über die Möglichkeiten und Grenzen der Maschinenverwendung zur Landschaftspflege. Die stärksten Schlegelgeräte werden auch mit stärkerem Holz fertig, die Arbeitsleistung wird dann aber zu gering. Wir sehen deshalb die Einsatzmöglichkeiten hauptsächlich in der Ginsterbekämpfung und in leichtem bis mittelstarkem Holzaufwuchs. Außerdem hoffen wir, möglichst viele Farnflächen in Zukunft maschinell mulchen zu können.

Die jetzt bestellte Maschine wird 135000.- DM kosten. Die Finanzierung erfolgt überwiegend durch die Landkreise und ist prozentual nach deren Anteil an den Gemeindeweiden aufgeteilt.

Die Kalkulation der Betriebskosten läßt sich wie folgt zusammenfassen:

- Betriebskosten je Einsatzstunde durchschnittlich 90.- DM, ohne Abschreibung (20.- DM/h).

- Produktive Arbeitszeit 6 Stunden pro Tag (+ 2 Stunden Transport und Wartung) - 130 Tage pro Jahr, also insgesamt 800 Stunden/Jahr.

- Einsatzleistung 0,5-2,5 ha/Tag $100-130 \mathrm{ha} / \mathrm{Jahr}$

\section{Abschließende Bemerkungen}

Es wurden hauptsächlich die im Südschwarzwald durchgeführten Landschaftspflegemaßnahmen, ihre Organisation und ihre Kosten behandelt. Die natürlichen Standortbedingungen und die agrarstrukturellen Verhältnisse wurden kaum erwähnt. Völlig unter den Tisch gefallen sind die rechtlichen und planerischen Voraussetzungen der dortigen Landschaftspflegemaßnahmen sowie das umfangreiche landschaftspflegerische Versuchsprogramm des Landes Baden-Württemberg, dessen Schwerpunkt ebenfalls im Südschwarzwald liegt.

\section{Literaturübersicht}

Deutsche Gesellschaft für Landentwicklung (DGL) (1974): Landschaftsrahmenplan Hinteres Renchtal, Stuttgart.

Deutsche Gesellschaft für Landentwicklung (DGL) (1957): Die Weidewirtschaft im südlichen Schwarzwald, Freiburg.

ENGEL, B. et al. (1973): Voraussichtliche Entwicklung der Landwirtschaft im Schwarzwald, Kemnat.

KETTLER, D. (1970): Landschaftsplan Feldberg/Schwarzwald, Schriftenreihe der Landesforstverwaltung Baden-Württ., Band 32, Stuttgart.
KETTLER, D. (1962): Über das Leistungspotential der Allmendweiden des Hochschwarzwaldes. In: Die Stoffproduktion der Pflanzendecke (Herausgeber H. Lieth), Stuttgart.

KETTLER, D. (1964): Großräumige Auswertung einer Vegetationskarte der Allmendweiden des Hochschwarzwaldes. In: Das wirtschaftseigene Futter, Heft 2, S. 101-111.

KETTLER, D. und FREI, J. (1965): Die Verbesserung der Allmendweiden im Südschwarzwald, dargestellt an der Gemeinde Schönenberg (Kr. Lörrach). In: Das wirtschaftseigene Futter, Heft 3, Seite 191-200.

Landkreis Lörrach (1969): Erhaltung der Landschaft und Verbesserung der Lebensbedingungen im Wiesental, Denkschrift, Lörrach.

Ministerium für Ernährung, Landwirtschaft, Weinbau und Forsten (1968): Schwarzwaldplan - Plan zur Ordnung und Verbesserung der besonderen Landeskultur in dem von Natur benachteiligten Gebiet des Schwarzwaldes, Stuttgart.

Ministerium für Ernährung, Landwirtschaft, Weinbau und Forsten (1972): Landwirtschafts- und Landeskulturgesetz, mit Anlage: Begründung zum Regierungsentwurf des Landwirtschafts- und Landeskulturgesetzes, Ges. BI. S. 74 (Sonderdruck), Stuttgart.

Ministerium für Ernährung, Landwirtschaft und Umwelt (1973): Schwarzwaldprogramm, Stuttgart.

MÜLLER, K. (Hrsg.) (1948): Der Feldberg im Schwarzwald Freiburg/Br., Freiburg.

MÜLLER, K. (1969): Der Schwarzwaldplan als Mittel zur Sicherung der Landbewirtschaftung und Beseitigung der Brache. In: "Der Schwarzwald», Heft 1, Freiburg.

MÜLLER, K. (1980): Der Schwarzwald als Agrarlandschaft. In: «Der Schwarzwald», Freiburg.

MÜLLER, K. (im Druck): Die Landwirtschaft im Belchengebiet. In: «Der Belchen».

Regierungspräsidium Südbaden (1969): Schwarzwaldplan Memorandum des Regierungspräsidiums Südbaden zur landwirtschaftlichen und gesamtwirtschaftlichen Entwicklung des Schwarzwaldes, Freiburg.

Regierungspräsidium Freiburg (1972): Modell eines Agrar- und Landschaftsplans dargestellt an der Gemeindegruppe Breitnau - St. Märgen - Waldau, Freiburg.

Regierungspräsidium Freiburg (1973): Modell II eines Agrarund Landschaftsplans dargestellt an der Gemeindegruppe Bernau - Menzenschwand - Präg, Freiburg.

RIES, H. (1948): Weide und Weidewirtschaft am Feldberg. In: Der Feldberg im Schwarzwald (Herausgeber K.Müller). Freiburg.

ROETHER, V. (1976): Landschaftsplan Feldberg/Schwarzwald II, Mitt. der Forstl. Versuchs- und Forschungsanstalt BadenWürttemberg, Heft 74, Freiburg.

SCHIEFER, J. (1981): Bracheversuche in Baden-Württemberg, Beihefte Veröffentlichungen Naturschutz und Landschaftspflege Baden-Württemberg Nr. 22, Karlsruhe.

SCHWABE-BRAUN, A. (1979): Weidfeldvegetation im Schwarzwald: Geschichte - Gesellschaften und ihre Komplexe Bedeutung für den Naturschutz, Dissertation, Freiburg.

WELLENDORF, A. (1930): Die Weidewirtschaft im Schwarzwald und Vorschläge zu ihrer Verbesserung. Schriftenreihe der Badischen Landwirtschaftskammer, Heft 14, Karlsruhe.

WILMANNS, O. und MÜLLER, K. (1976): Beweidung mit Schafen und Ziegen als Landschaftspflegemaßnahmen im Schwarzwald? In: Natur und Landschaft, Heft 10, S. 271-274, Stuttgart. 